\title{
Incidence and prevalence trends of youth-onset type 2 diabetes in a cohort of Canadian youth: 2002-2013
}

\author{
Shazhan Amed ${ }^{1}$, Nazrul Islam ${ }^{2,3}$, Jenny Sutherland ${ }^{4}$, Kim Reimer $^{4}$ \\ ${ }^{1}$ Department of Pediatrics, University of British Columbia, Vancouver, BC, Canada \\ ${ }^{2}$ MRC Epidemiology Unit, University of Cambridge, Cambridge, UK \\ ${ }^{3}$ Harvard T.H. Chan School of Public Health, Boston, MA, USA \\ ${ }^{4}$ BC Ministry of Health, Population Health Surveillance \& Epidemiology, Victoria, BC, Canada
}

Corresponding author:

Dr Shazhan Amed, Department of Pediatrics, University of British Columbia, K4-206, 4480 Oak Street, Vancouver, British Columbia V6H 3V4, Canada. Email: samed@cw.bc.ca

\begin{abstract}
Objective: Youth-onset type 2 diabetes is an emerging disease. We estimated incidence and prevalence trends of youth-onset type 2 diabetes between 2002 and 2013 in the Canadian province of British Columbia.

Methods: This population-based cohort study used a validated diabetes case-finding definition and algorithm to differentiate type 2 from type 1 diabetes to identify youth $<20$ years with type 2 diabetes within linked populationbased administrative data. Age-standardized incidence and prevalence were calculated. JoinPoint regression and double exponential smooth modeling were used.

Results: From 2002/03-2012/13, the incidence of youth-onset type 2 diabetes increased from 3.45 (95\% CI: 2,43, 4.80 ) to 5.16 (95\% CI: 3.86, 6.78)/100,000. The annual percent change (APC) in incidence was 3.74 (95\% CI: 1.61, 5.92; $\mathrm{p}=0.003$ ) overall, while it was 5.94 (95\% CI: 1.84, 10.20; p=0.009) and 0.53 (95\% CI: -5.04, 6.43; p=0.837) in females and males, respectively. The prevalence increased from 0.009\% (95\% CI: 0.007, 0.011) in 2002/03 to 0.021\% (95\% CI: 0.018, 0.024) in 2012/13 with an APC of 7.89 (95\% CI: 6.41, 9.40; $p<0.0001)$. In females, it increased from $0.012 \%$ (95\% CI: $0.009,0.015)$ to $0.027 \%$ (95\% CI: $0.023,0.032)$ and in males from $0.007 \%$ (95\% CI: $0.005,0.009$ ) to $0.015 \%$ (95\% CI: 0.012, 0.019). By 2030, we forecast a prevalence of $0.046 \%$ [95\% CI: 0.043 , 0.048].
\end{abstract}

Conclusions: Youth-onset type 2 diabetes is increasing with higher rates in females versus males. If these rates continue, in 2030, the number of cases will increase by 5-fold. These data are needed to set priorities for diabetes prevention in youth.

MeSH Key Words: type 2 diabetes mellitus; epidemiology; incidence; prevalence; pediatrics 


\section{INTRODUCTION}

Incidence rates of youth-onset type 2 diabetes vary worldwide. In the United States (2007), annual incidence rate was 9.0 cases per 100,000 youth 0-19 years of age per year, (1), while in Canada (2006-2008), the annual minimum incidence rate was 1.54 cases per 100,000 youth <18 years of age (2). The U.K and other European countries report much lower rates of youth-onset type 2 diabetes $(3,4)$. Although the absolute number of cases of youth-onset type 2 diabetes appear inconsequential when compared to the number of cases in adults, the natural history of this emerging disease suggests youth-onset type 2 diabetes will significantly impact the health of children and youth today, and for generations to come.

Youth-onset type 2 diabetes is materializing to be a more severe disease than adult-onset type 2 diabetes. As shown by the TODAY Study Group that conducted a clinical trial in the U.S to maintain glycemic control in youthonset type 2 diabetes, diabetes-related complications develop quickly $(5,6)$ and are often present at diagnosis (2). SEARCH reported that, when compared to youth with type 1 diabetes, those with type 2 diabetes have a significantly increased higher odds of developing diabetic kidney disease, retinopathy, and peripheral neuropathy (7). Also, treatment of youth-onset type 2 diabetes with oral hypoglycemic agents is largely ineffective and almost $50 \%$ of cases require subcutaneous insulin because of metabolic decompensation (8). Mortality is also higher in those who develop type 2 diabetes at a young age where an inverse relationship between age of diabetes onset and standardized mortality ratio (SMR) has been reported with the highest SMR (3.4 [95\% CI 2.7-4.2]) in those diagnosed with diabetes between 15 and 30 years of age (9).

Evidence suggests an increase in both incidence and prevalence of youth-onset type 2 diabetes, albeit at varying degrees depending on the population demographic. In the U.S., the overall prevalence of type 2 diabetes in youth $<20$ years increased by 30.5\% between 2001 and 2009 (10), with predictions that the prevalence will increase by $178 \%$ by 2050 (11). Incidence rates are also on the rise in the U.S with an estimated relative increase of $4.8 \%$ (95\% CI: 3.2, 6.4; $\mathrm{p}<0.001$ ) per year between 2002/03 to 2011/12 (1).

In Canada, administrative health data have been used to describe epidemiological trends of diabetes in youth (12), however, type 1 and type 2 diabetes are for the most part reported separately and therefore trends are assumed to reflect type 1 diabetes, the most common form of childhood diabetes. We have developed a validated algorithm for differentiating between type 1 and type 2 diabetes in administrative datasets using demographic and drug utilization data (13) and have recently reported incidence and prevalence trends of childhood type 1 diabetes (14). The objective of this study was to describe trends in the incidence and prevalence of youth-onset type 2 diabetes in a population-based sample of Canadian youth $<20$ years of age. 


\section{METHODS}

\section{Data source:}

We used four datasets that exist within a universal health care system in the Canadian province of British Columbia. These databases are linkable using an individual's personal health number (PHN) and include: (i) client registration information (Client Registry \& Premium Billing); (ii) physician fee-for-service claims for outpatient services (Medical Services Plan - MSP); (iii) health claims for hospital admissions (Discharge Abstract Database DAD); and (iv) provincial prescription dispensations (PharmaNet). To establish the Diabetes Database, a diabetes case-finding definition was applied to the linked data where an individual with any 1 of following within a 1-year period was included: 1 hospital discharge code for diabetes; 2 outpatient physician MSP claims coded for diabetes; 2 or more prescription dispensations for insulin; 2 or more prescription dispensations for an oral anti-diabetic medication (except metformin); or two of more of: 1 prescription dispensation for insulin, 1 prescription dispensation for an oral anti-diabetic medication, 2 prescriptions for metformin and 1 outpatient physician MSP claim coded as diabetes. Once individuals were included in the Diabetes Database, they remained in it until they moved out of the province or died. For the purpose of this study, data was retrieved on individuals $<20$ years of age within the Diabetes Database. The diabetes case-finding definition used to create the Diabetes Database has been shown to be highly sensitive (97\%) in the $<20$ year old population when validated against a clinical database comprised of physician-diagnosed pediatric type 1 and type 2 diabetes (15).

\section{Study population and period:}

In this population-based study examining an average of 950,000 individuals $<20$ years of age each year, we included all individuals $>1$ year of age and $<20$ years of age in the Diabetes Database between April $1^{\text {st }}$, 2002 to March $31^{\text {st }}$, 2013 who were classified as having type 2 diabetes within the province of British Columbia. A case was classified as type 2 diabetes if they were 10 years of age or older, and their prescription dispensations within 2 years (730 days) of meeting the case-finding algorithm (the index date) included an oral anti-diabetic medication (i.e. metformin, sulfonylurea) alone or in combination with insulin, \pm glucose strips. This diabetes differentiating algorithm was $83.2 \%$ sensitive and $97.5 \%$ specific in classifying cases of type 2 diabetes in individuals $<20$ years of age, with a positive predictive value of $73.7 \%$, when compared to a gold standard clinical database of physiciandiagnosed pediatric type 1 and type 2 diabetes (13). Individuals who did not show evidence of diabetes-related prescription dispensations within 2 years (730 days) of their index date were excluded from the analysis (Figure 1).

\section{Definition of main outcomes:}

We identified incident and prevalent cases for each fiscal year (April $1^{\text {st }}$ to March $31^{\text {st }}$ ) from 2002 to 2013 , based on the following definitions. Incident cases: (i) the individual met the conditions of the diabetes case-finding algorithm for the first time, and the date at which this occurred was the index date for diagnosis; and (ii) the individual met the conditions of the diabetes differentiating algorithm as a case of type 2 diabetes. Prevalent cases: 
cases that existed at the beginning of that fiscal year that met the conditions of the diabetes differentiating algorithm as a case of type 2 diabetes, in addition to incident cases that were identified during that year.

\section{Statistical analysis:}

Age-standardized incidence (per 100,000) and prevalence rates (per 100) were calculated using 1990/91 Canadian population as the standard population and Census data (StatsCan) on the BC population $<20$ years of age. The JoinPoint regression analysis program (V4.3.1.0) was used to examine the trends in age-standardized incidence and prevalence rates, overall, and by gender. We used the permutation test (with 5000 randomly permuted datasets) method in the Joinpoint program that selects the best fitting piecewise continuous log-linear model (16) at a significance level of 0.05 to calculate the annual percent change (APC) with corresponding 95\% confidence intervals. We also used double exponential smooth modeling in SAS [9.4] to project the number and prevalence rates of type 2 diabetes until 2030.

\section{RESULTS}

Incidence of youth-onset type 2 diabetes:

In 2002/03, 37 (62\% female) new cases of youth-onset type 2 diabetes were identified increasing to 53 (68\% female) new cases in 2012/13. Incidence was 3.45 (95\% CI: 2.43, 4.80) per 100,000 in 2002/03 and 5.16 (95\% CI: 3.86, 6.78) per 100,000 in 2012/13. During the same time period, incidence increased from 4.43 (95\% CI: 2.81, 6.74) to 7.21 (95\% CI: $5.05,10.05)$ in females and from 2.53 (95\% CI: $1.39,4.36)$ to 3.23 (95\% CI: 1.88, 5.24) in males (Table 1 - online). The sex differences in type 2 diabetes incidence widened after 2010 (Figure 2A).

The APC in incidence was 3.74 (95\% CI: 1.61, 5.92; p=0.003) overall, while it was 5.94 (95\% CI: 1.84, 10.20; $\mathrm{p}=0.009$ ) and 0.53 (95\% CI: $-5.04,6.43 ; \mathrm{p}=0.837)$ in females and males, respectively. While the overall APC did not increase in males, there was a marginally significant increase in the APC between 2002-2007 (APC: 11.95, 95\% CI: 0.15, 25.14; p=0.048), and a marginally significant decrease between 2007-2012 (APC: -9.62, 95\% CI: 18.7, 0.49; $\mathrm{p}=0.058$ ). The APC in the overall population was the highest from 2002-2008 (APC: 6.35, 95\% CI: 2.31, 10.55; $\mathrm{p}=0.008$ ). Incidence was highest among females (Figure 3C) and youth aged 15-19 years (Figure 3D) where it increased from 10.57 (95\% CI: 7.13, 15.09) in 2002/03 to 13.81 (95\% CI: 9.82, 18.88) in 2012/13 (Table 1 online).

\section{Prevalence of youth-onset type 2 diabetes:}

From 2002/03 to 2012/13, prevalent cases increased from 97 (63\% female) to 219 (63\% Female) and prevalence increased from $0.009 \%$ (95\% CI: $0.007,0.011)$ to $0.021 \%$ (95\% CI: $0.018,0.024$ ) representing a 2.3-fold increase over a decade. In the same time period, prevalence increased from $0.012 \%$ (95\% CI: $0.009,0.015)$ to $0.027 \%$ (95\% CI: $0.023,0.032$ ) in females, and from $0.007 \%$ (95\% CI: $0.005,0.009)$ to $0.015 \%$ (95\% CI: 0.012 , 0.019 ) in males (Table 2 -online). 
The APC in the overall prevalence was 7.89 (95\% CI: 6.41, 9.40; $\mathrm{p}<0.0001$ ), while the same for females and males was 7.98 (95\% CI: 6.94, 9.02; $\mathrm{p}<0.0001$ ) and 7.77 (95\% CI: 4.09, 11.57; p=0.001), respectively. Prevalence increased at a much higher rate from 2002-2008 (APC: 10.84, 95\% CI: 8.64, 13.08, p<0.0001) followed by a slower increase thereafter (APC: 3.86, 95\% CI: 0.80, 7.0, p=0.021). A significant increase in prevalence was also found among females aged 10-14 years.

Projections of youth-onset type 2 diabetes prevalence through 2020:

Assuming similar rates and APC, the projected total number of prevalent cases of youth-onset type 2 diabetes will increase to 350 (95\% CI: 326, 374) in 2020, 421 (95\% CI: 394, 447) in 2025 and 491 (95\% CI: 462, 519) in 2030, representing more than a 5-fold increase in the number of prevalent cases of type 2 diabetes in children since 2002/03. The prevalence will increase to $0.046 \%$ (95\% CI: 0.043, 0.048) by 2030 (Figure 4).

\section{DISCUSSION}

To our knowledge, this study is the first of its kind to use administrative health data to describe trends in youth-onset type 2 diabetes. We report an increasing trend in the incidence and prevalence of youth-onset type 2 diabetes over the last decade in a population-based sample of Canadian youth, with distinct sex differences where young females are affected more than males. The majority of the burden of youth-onset type 2 diabetes was in the 15-19 year age bracket although, an increasing trend in prevalence among females aged 10-14 years was also observed. If current rates continue, the prevalence of youth-onset type 2 diabetes will increase by 5 -fold by the year 2030.

A similar increase in youth-onset type 2 diabetes has been reported in the U.S. and among First Nation youth in Canada. The SEARCH study reported that overall prevalence of youth-onset type 2 diabetes in the U.S rose from $0.034 \%$ in 2001 to $0.046 \%$ in 2009 , representing a 35\% relative increase when adjusted for differences in completeness of ascertainment (10). In the same U.S population, unadjusted incidence rate of youth-onset type 2 diabetes increased by 7.1\% annually ( $<<0.001$ ) from 2002/03 to 2011/12 (1). In Canadian First Nation youth $<18$ years of age, the incidence of type 2 diabetes doubled over a 5-year period (9.03 to 20.58 cases per 100,000 youth per year) (17). European data shows little or no increase in youth-onset type 2 diabetes (18) however, surveillance has typically excluded youth aged 15-19 years, the age bracket that faces the highest burden of disease. Austria recently reported a stable incidence of type 2 diabetes in youth $\leq 15$ years of age from 1999 to 2015 (4).

The Canadian Diabetes Association first published clinical practice guidelines on the management of youthonset type 2 diabetes in 2003 with updates in 2008 and 2013. Our data showed the highest incidence rate (5.85 cases per 100,000/year) in 2008 and the highest APC (6.35) from 2002-2008, suggesting that increased awareness of and screening for youth-onset type 2 diabetes has contributed to the increasing epidemiological trends. Rising rates of overweight and obesity also likely contribute to this finding. The prevalence of overweight and obesity doubled among Canadians aged 12-17 years over 25 years (1978/79-2004), and obesity alone tripled (19). In a study of Canadian youth with new-onset type 2 diabetes, 95\% were obese and the remaining were overweight, confirming 
obesity as a key risk factor for youth-onset type 2 diabetes (2). Recent data suggest a decline in the prevalence of overweight and obesity in Canadian children from 30.7\% to 27\% ( $p<0.001)$ from 2009 to 2013 (20), which may translate into declining trends of youth-onset type 2 diabetes in the future. Recent data released by Statistics Canada comparing rates of childhood obesity in 2004 versus 2015 showed that in the province of British Columbia, obesity rates decreased more in males (16.8\% in 2004 to $11.1 \%$ in 2015) than in females (11\% in 2004 to $9.1 \%$ in 2015) (StatsCan - http://www5.statcan.gc.ca), possibly explaining the sex differences in incidence trends of youth-onset type 2 diabetes in our region.

Our data show a 3:1 female predominance for youth-onset type 2 diabetes in 2012/13, and a widening gap in incidence between males and females since 2005. These sex differences have been described in the literature, and across all ethnic groups $(10,17,21)$. Interestingly, in adults, the opposite has been reported; globally, 15.6 million more men than women had diabetes in 2015 (22). There continues to be a considerable gap in knowledge on the complex interplay of hormones, genes, lifestyle and environment that might contribute to the sex differences in rates of youth-onset type 2 diabetes, and why this trend reverses in adulthood. Insulin resistance during puberty can be as much as $30 \%$ greater in girls compared to boys (23) and a recent study by Cooper et al showed that pubertal females have a 30-40\% higher insulinaemic response to a high glycemic index meal compared to pubertal males (24). These sex differences may partly be explained by higher adiposity in pubertal females versus males $(25,26)$. Increased prevalence of youth-onset type 2 diabetes in females 15 years of age and older may also be due to more frequent screening and/or recognition of type 2 diabetes as part of the pre-natal medical assessment. Lastly, our data may have inadvertently captured young females with polycystic ovarian syndrome treated with metformin where the physician incorrectly used a diabetes diagnostic code.

Higher rates of type 2 diabetes in females entering child-bearing age has significant implications and requires an immediate public health response. The TODAY trial reported on female youth with type 2 diabetes (mean diabetes duration of 3 years) who became pregnant ( $\mathrm{N}=53$ pregnancies). Among these pregnancies, almost 25\% resulted in fetal loss and 2 ended in stillbirth. Of the pregnancies resulting in a live-born infant, 15\% were preterm and $20 \%$ had a major congenital anomaly (27). There is also strong evidence supporting that fetal over-nutrition and a hyperglycemic intrauterine environment increases the risk of obesity and early-onset type 2 diabetes in the offspring, resulting in a vicious cycle that may be contributing to increasing rates of youth-onset type 2 diabetes (28). In Canada, incidence rates of both gestational and pre-gestational diabetes doubled from 1996-2010 in women aged 15-50 years (29). Rising rates of youth-onset type 2 diabetes will add to this increasing burden of diabetes in pregnancy.

This study has several strengths: it is population-based, includes 11 years of data, and is the first to differentiate between type 2 from type 1 diabetes in administrative data enabling the delineation of epidemiological trends for youth-onset type 2 diabetes at a population level. Limitations of our study include the inability to describe trends by ethnicity and the potential for misclassifying cases of type 2 diabetes as type 1 diabetes and vice versa. The absence of clinical information within administrative data such as pancreatic antibody levels makes 
classification of diabetes type more challenging. Also, in a validation study using a combination of electronic medical record (EMR) and administrative data to identify and classify youth with diabetes, $9 \%$ of youth with physician diagnosed type 1 diabetes used metformin and insulin (30). In our study, these children would be misclassified as type 2 diabetes, potentially overestimating our rates. However, in our previous validation research, algorithms to differentiate type 1 vs. type 2 diabetes that classified insulin plus metformin users as type 1 diabetes performed less favorably compared to algorithms that classified this drug utilization pattern as type 2 diabetes (13). Our algorithm also assumed that individuals under 10 years of age had type 1 diabetes, potentially underestimating type 2 diabetes rates because, although type 2 diabetes under 10 years of age is uncommon, in some studies, it has accounted for roughly $10 \%$ of youth-onset type 2 diabetes cases $(2,17)$. Children with existing type 2 diabetes that moved to BC from another province would have been counted as an incident (rather than a prevalent) case because we do not have access to data from other Canadian provinces. Lastly, our forecasting model, although similar to our observed data, should be interpreted with caution as it is based on only 11 years of data, assumes no change in clinical practice that could result in the identification of more (or less) cases, and does not take into account the recent data indicating decreasing rates of overweight and obesity in Canadian children (StatsCan).

Youth-onset type 2 diabetes is increasing at an alarming rate and if prevention efforts are not given priority among public health officials, the number of cases in British Columbia, Canada will increase by 5 -fold by the year 2030. British Columbia has some of the lowest rates of childhood obesity and overweight in Canada, and therefore, rates in other provinces are likely at least similar, if not higher than what is reported here. The greatest burden of youth-onset type 2 diabetes is in older youth aged 15-19 years who likely access primary, rather than pediatric care. Therefore, increasing awareness of youth-onset type 2 diabetes among primary care practitioners is critical to ensure early identification and initiation of management so as to prevent serious diabetes-related complications and early mortality. The female predominance is particularly concerning considering the impact of maternal obesity and diabetes during pregnancy on the metabolic health of future offspring. Our findings will inform resource allocation for health services and public health, and ongoing surveillance will help to assess the impact of diabetes prevention initiatives designed for youth. Further research is needed to examine factors contributing to rising rates of youthonset type 2 diabetes, as well as the pathophysiological and environmental mechanisms leading to the observed sex differences.

Funding: No funding was received for this study.

Conflict of interest: The authors have no potential, perceived or real conflicts to disclose.

Author contributions: S.A. designed the study, researched/interpreted the data and wrote the manuscript. K.R and J.S mined and analyzed the data, contributed to the discussion and reviewed/edited the manuscript. N.I conducted trend analyses and forecasting, contributed to the discussion and reviewed/edited the manuscript. This study did not receive funding. An honorarium, grant, or other form of payment was not provided to any author to produce the manuscript. 


\section{REFERENCES:}

1. Mayer-Davis EJ, Lawrence JM, Dabelea D, Divers J, Isom S, Dolan L, et al. Incidence Trends of Type 1 and Type 2 Diabetes among Youths, 2002-2012. N Engl J Med. 2017 Apr 13;376(15):1419-29.

2. Amed S, Dean HJ, Panagiotopoulos C, Sellers EAC, Hadjiyannakis S, Laubscher TA, et al. Type 2 diabetes, medication-induced diabetes, and monogenic diabetes in Canadian children: a prospective national surveillance study. Diabetes Care. 2010 Apr;33(4):786-91.

3. Haines L, Wan KC, Lynn R, Barrett TG, Shield JPH. Rising incidence of type 2 diabetes in children in the U.K. Diabetes Care. 2007 May;30(5):1097-101.

4. Abstracts for the 42nd Annual Meeting of the International Society for Pediatric and Adolescent Diabetes (ISPAD), 26-29 October 2016, Valencia, Spain. Pediatric Diabetes. 2016 Oct;17 Suppl 24:5-176.

5. TODAY Study Group. Retinopathy in Youth With Type 2 Diabetes Participating in the TODAY Clinical Trial. Diabetes Care. 2013 Jun;36(6):1772-4.

6. TODAY Study Group. Rapid Rise in Hypertension and Nephropathy in Youth With Type 2 Diabetes: The TODAY clinical trial. Diabetes Care. 2013 Jun;36(6):1735-41.

7. Dabelea D, Stafford JM, Mayer-Davis EJ, D'Agostino R, Dolan L, Imperatore G, et al. Association of Type 1 Diabetes vs Type 2 Diabetes Diagnosed During Childhood and Adolescence With Complications During Teenage Years and Young Adulthood. JAMA. 2017 Feb 28;317(8):825-35.

8. TODAY Study Group, Zeitler P, Hirst K, Pyle L, Linder B, Copeland K, et al. A clinical trial to maintain glycemic control in youth with type 2 diabetes. N Engl J Med. 2012 Jun 14;366(24):2247-56.

9. Al-Saeed AH, Constantino MI, Molyneaux L, D’Souza M, Limacher-Gisler F, Luo C, et al. An Inverse Relationship Between Age of Type 2 Diabetes Onset and Complication Risk and Mortality: The Impact of Youth-Onset Type 2 Diabetes. Diabetes Care. 2016 Apr 21;39(5):823-9.

10. Dabelea D, Mayer-Davis EJ, Saydah S, Imperatore G, Linder B, Divers J, et al. Prevalence of Type 1 and Type 2 Diabetes Among Children and Adolescents From 2001 to 2009. JAMA. 2014 May 7;311(17):1778.

11. Imperatore G, Boyle JP, Thompson TJ, Case D, Dabelea D, Hamman RF, et al. Projections of Type 1 and Type 2 Diabetes Burden in the U.S. Population Aged. Diabetes Care. 2012 Dec;35(12):2515-20.

12. Public Health Agency of Canada. Diabetes in Canada: Facts and figures from a public health perspective. 2011. https://www.canada.ca/en/public-health/services/chronic-diseases/reportspublications/diabetes/diabetes-canada-facts-figures-a-public-health-perspective.html [Last Accessed August $11,2017]$

13. Vanderloo SE, Johnson JA, Reimer K, McCrea P, Nuernberger K, Krueger H, et al. Validation of classification algorithms for childhood diabetes identified from administrative data. Pediatric Diabetes. 2012 May;13(3):229-34.

14. Fox D, Islam N, Sutherland JE, Reimer K, Amed S. Type 1 Diabetes Incidence and Prevalence Trends in a Cohort of Canadian Children and Youth. Pediatric Diabetes. 2017 [Epub Ahead of Print]

15. Amed S, Vanderloo SE, Metzger D, Collet J-P, Reimer K, McCrea P, et al. Validation of diabetes case definitions using administrative claims data. Diabetic Medicine. 2011 Apr;28(4):424-7.

16. Kim HJ, Fay MP, Feuer EJ, Midthune DN. Permutation tests for joinpoint regression with applications to cancer rates. Stat Med. 2000 Feb 15;19(3):335-51. 
17. Sellers E, Wicklow BA, Dean HJ. Clinical and demographic characteristics of type 2 diabetes in youth at diagnosis in Manitoba and northwestern Ontario (2006-2011). Canadian Journal of Diabetes. Canadian Journal of Diabetes. 2012 36:114-118.

18. Schober E, Waldhoer T, Rami B, Hofer S, Austrian Diabetes Incidence Study Group. Incidence and time trend of type 1 and type 2 diabetes in Austrian children 1999-2007. The Journal of Pediatrics. 2009 Aug;155(2):190-1.

19. Shields M. Overweight and obesity among children and youth. Health Reports. 2006. 17:27-42.

20. Rodd C, Sharma AK. Recent trends in the prevalence of overweight and obesity among Canadian children. Canadian Medical Association Journal. 2016. 188:313-320.

21. Cai M, Kappelman MD, Girman CJ, Jain N, Stürmer T, Brookhart MA. Trends and Determinants of Oral Anti-Diabetic Initiation in Youth with Suspected Type 2 Diabetes. Meyre D, editor. PLoS ONE. 2015 Oct 28;10(10):e0140611-3.

22. Federation ID. IDF diabetes atlas. 2011. http://www.diabetesatlas.org [Last Accessed August 2017]

23. Metcalf BS, Hosking J, Henley WE, Jeffery AN, Mostazir M, Voss LD, et al. Physical activity attenuates the mid-adolescent peak in insulin resistance but by late adolescence the effect is lost: a longitudinal study with annual measures from 9-16 years. Diabetologia. 2nd ed. 2015 Aug 12;58(12):2699-708.

24. Cooper SB, Dring KJ, Morris JG, Cousins BEW, Nute ML, Nevill ME. Sex differences in adolescents' glycaemic and insulinaemic responses to high and low glycaemic index breakfasts: a randomised control trial. Br J Nutr. Cambridge University Press; 2017 Feb;117(4):541-7.

25. Jeffery AN, Metcalf BS, Hosking J, Streeter AJ. Age before stage: insulin resistance rises before the onset of puberty. Diabetes. 2012 35:536-541.

26. Araújo J, Barros H, Severo M, Lopes C, Ramos E. Longitudinal changes in adiposity during adolescence: a population-based cohort. BMJ Open. British Medical Journal Publishing Group; 2014 Jun 10;4(6):e0043800 .

27. Klingensmith GJ, Pyle L, Nadeau KJ, Barbour LA, Goland RS, Willi SM, et al. Pregnancy Outcomes in Youth With Type 2 Diabetes: The TODAY Study Experience. Diabetes Care. 2015 Dec 22;39(1):122-9.

28. Dabelea D, Crume T. Maternal Environment and the Transgenerational Cycle of Obesity and Diabetes. Diabetes. 2011 Jun 27;60(7):1849-55.

29. Feig DS, Hwee J, Shah BR, Booth GL, Bierman AS, Lipscombe LL. Trends in incidence of diabetes in pregnancy and serious perinatal outcomes: a large, population-based study in Ontario, Canada, 1996-2010. Diabetes Care. 2014 Jun;37(6):1590-6.

30. Zhong VW, Pfaff ER, Beavers DP, Thomas J, Jaacks LM, Bowlby DA, et al. Use of administrative and electronic health record data for development of automated algorithms for childhood diabetes case ascertainment and type classification: the SEARCH for Diabetes in Youth Study. Pediatric Diabetes. 2014 Dec;15(8):573-84. 
Figure 1: Classification of Prevalent Cases of Diabetes in Individuals $<20$ years (2012/13)

$$
\begin{aligned}
& \text { Individuals }>1 \text { years of age and }<20 \text { years of age } \\
& \text { meeting the case finding algorithm for diabetes } \\
& \text { between April } 1^{\text {st }}, 2002 \text { to March } 31^{\text {st }}, 2013
\end{aligned}
$$

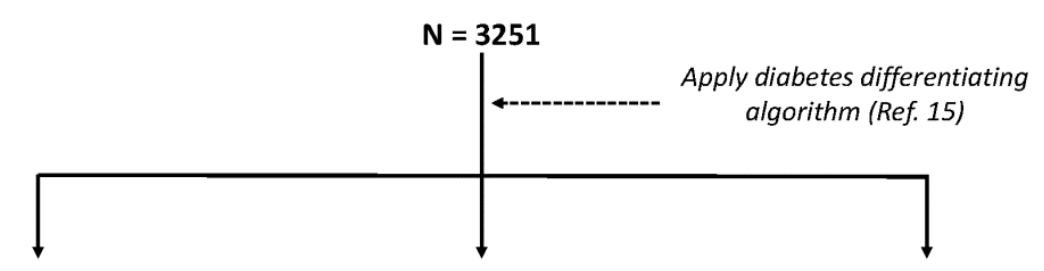

Individuals $<10$ years $O R$ $>10$ years and using insulin \pm strips only

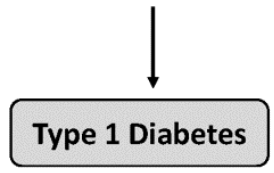

$\mathbf{N}=\mathbf{2 2 6 4}$
Individuals $>10$ years and $<20$ years with no evidence of drug utilization within 2 years of index date

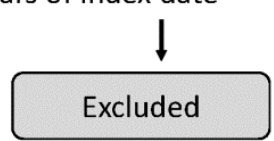

$N=768$
Individuals $>10$ years and $<20$

years with evidence of insulin and oral anti-diabetic medication utilization in PharmaNet

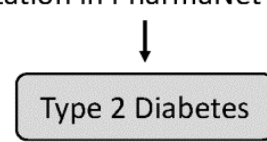

$\mathbf{N}=\mathbf{2 1 9}$ 

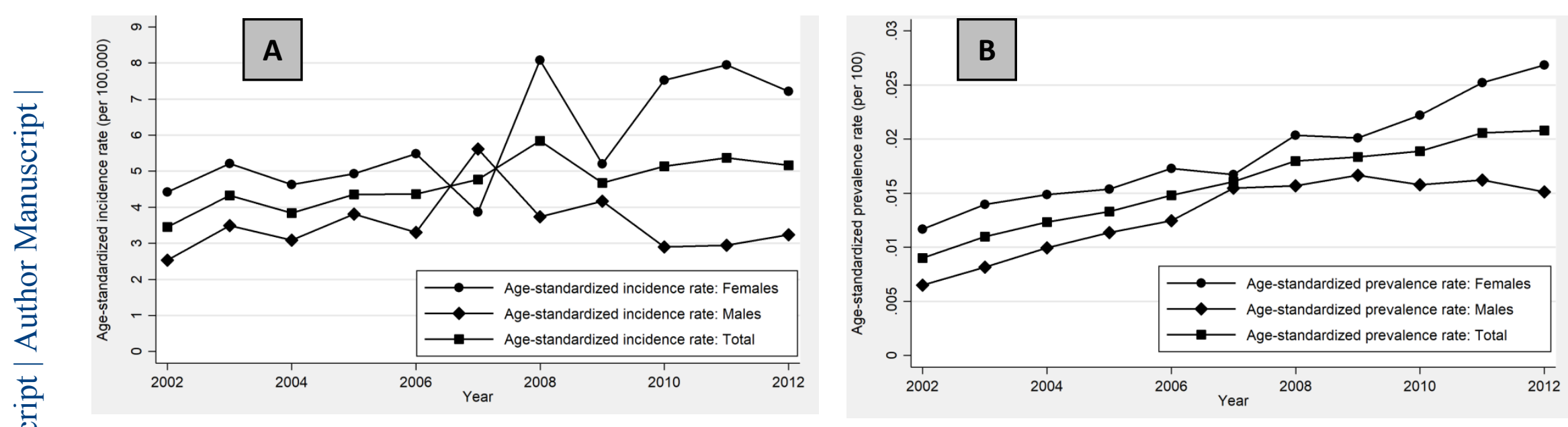

Figure 2: Age standardized incidence (A) and prevalence (B) rates by sex and year 


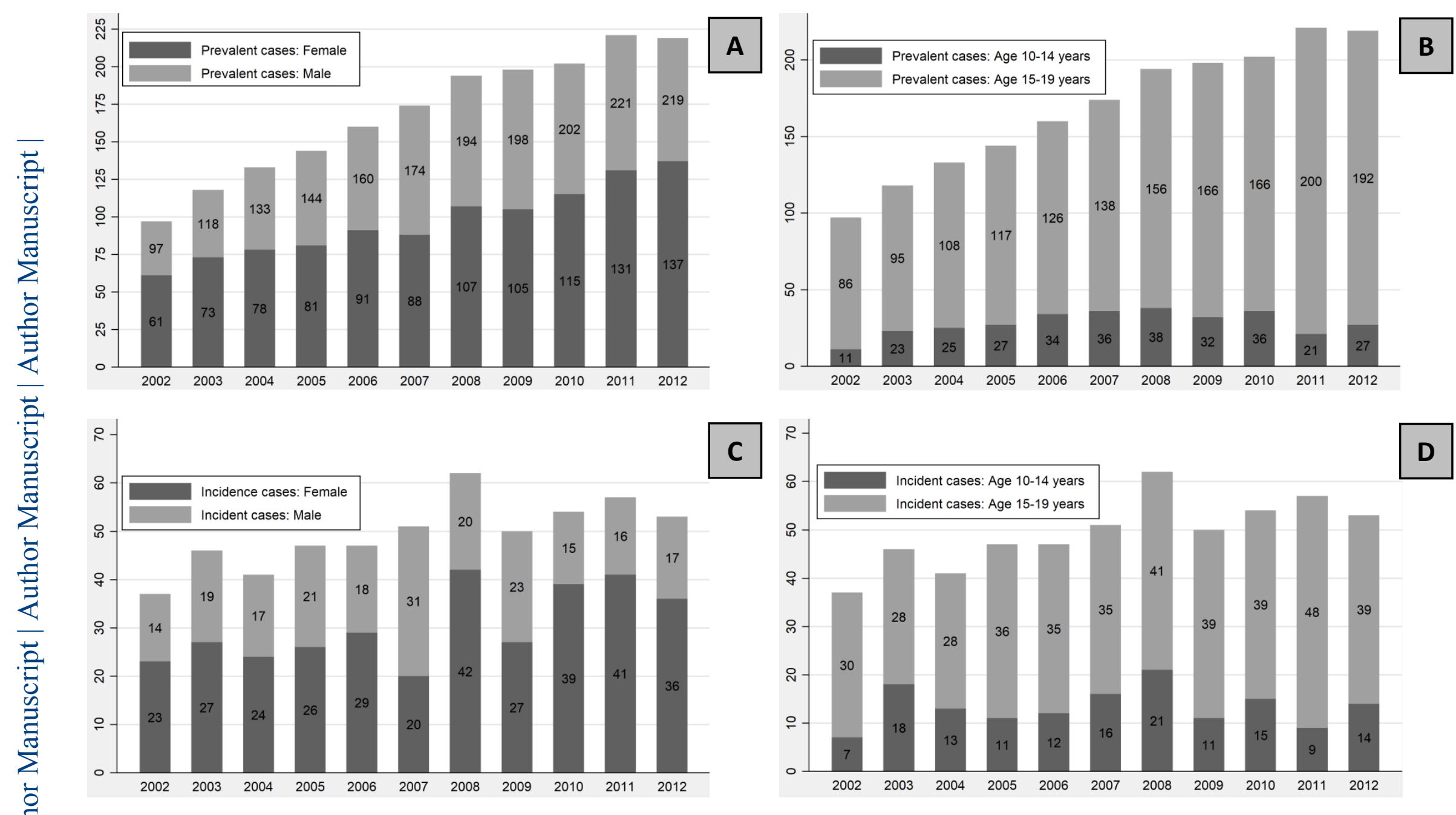

Figure 3: Incident and prevalent cases by sex and age group 


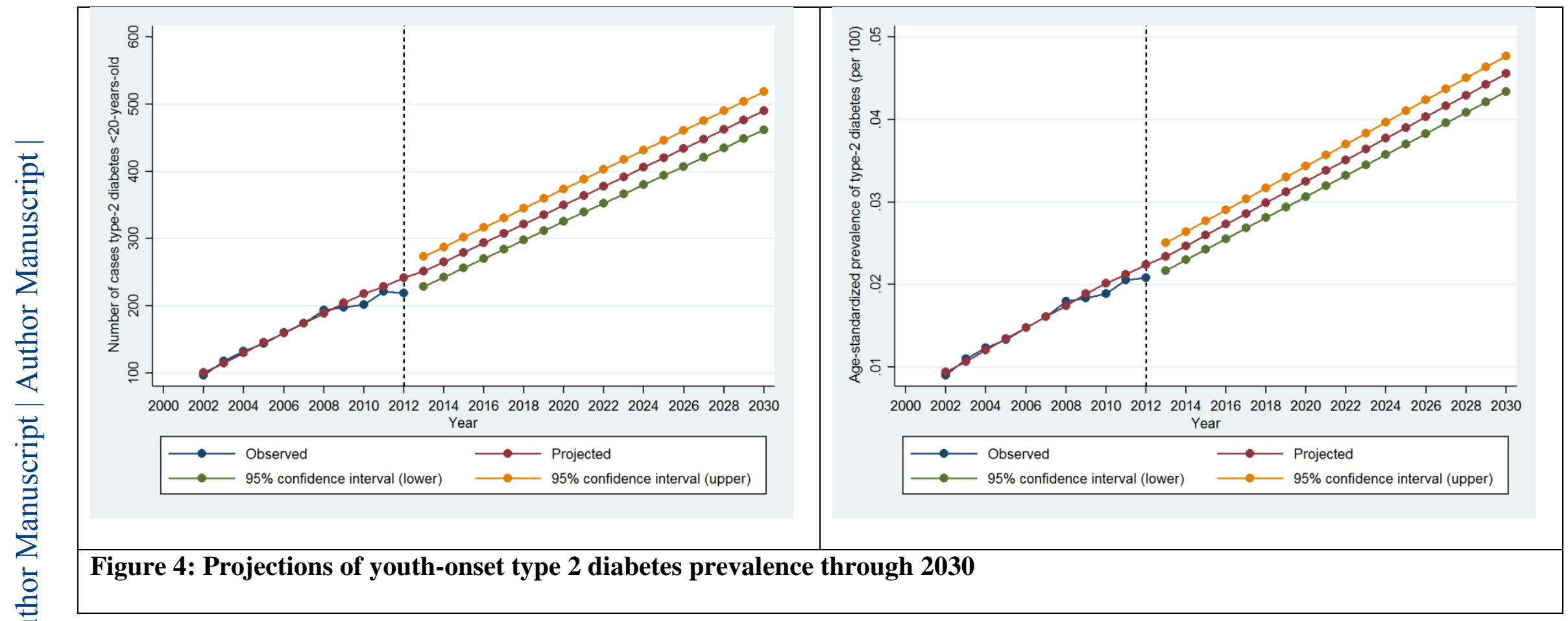




\section{Supplementary Materials}

Table 1; online only: Age-Standardized and Age-Specific Incidence Rates (per 100,000 [95\% CI]) of T2D in youth 10-19 years of age

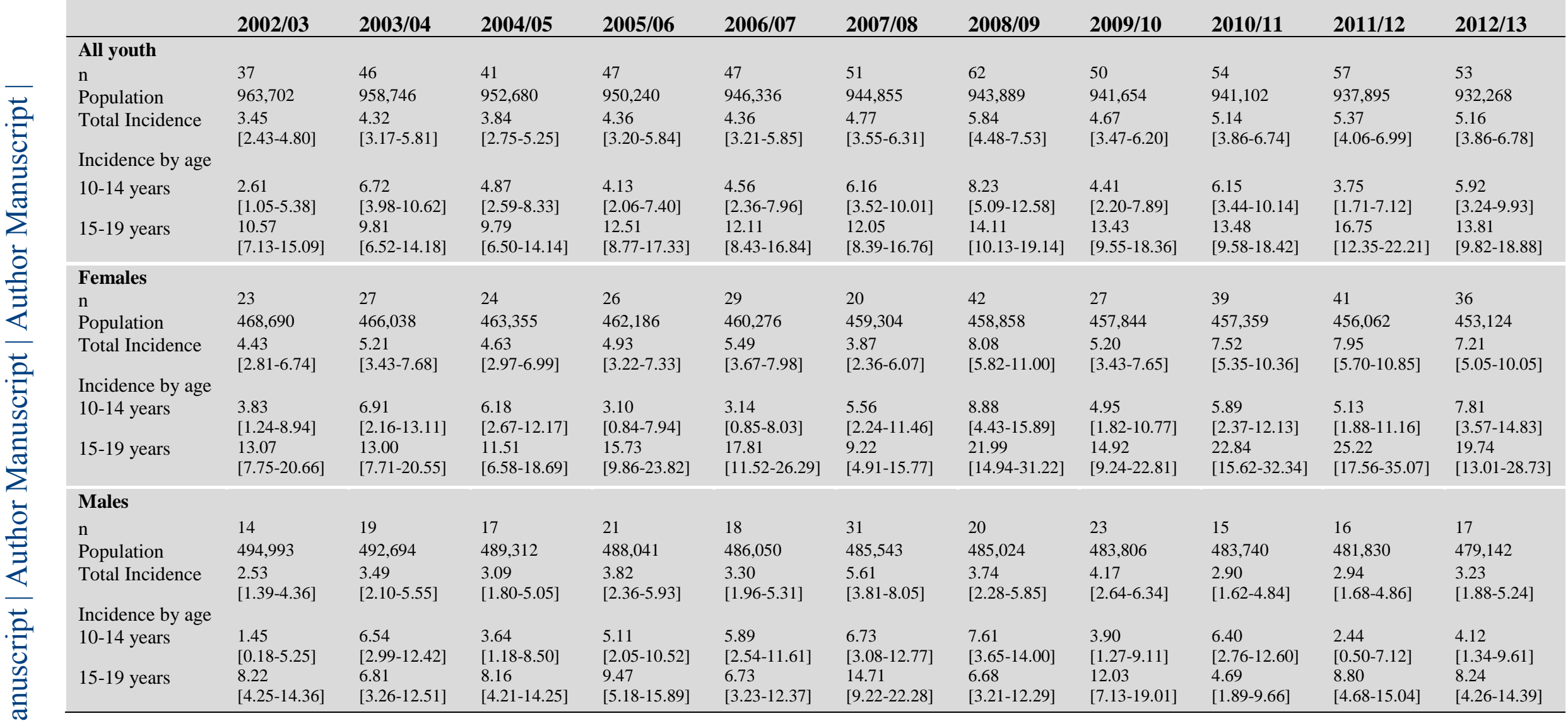


Table 2; online only: Age-Standardized and Age-Specific Prevalence (per 100) [95\% CI] of T2D in youth 10-19 years of age

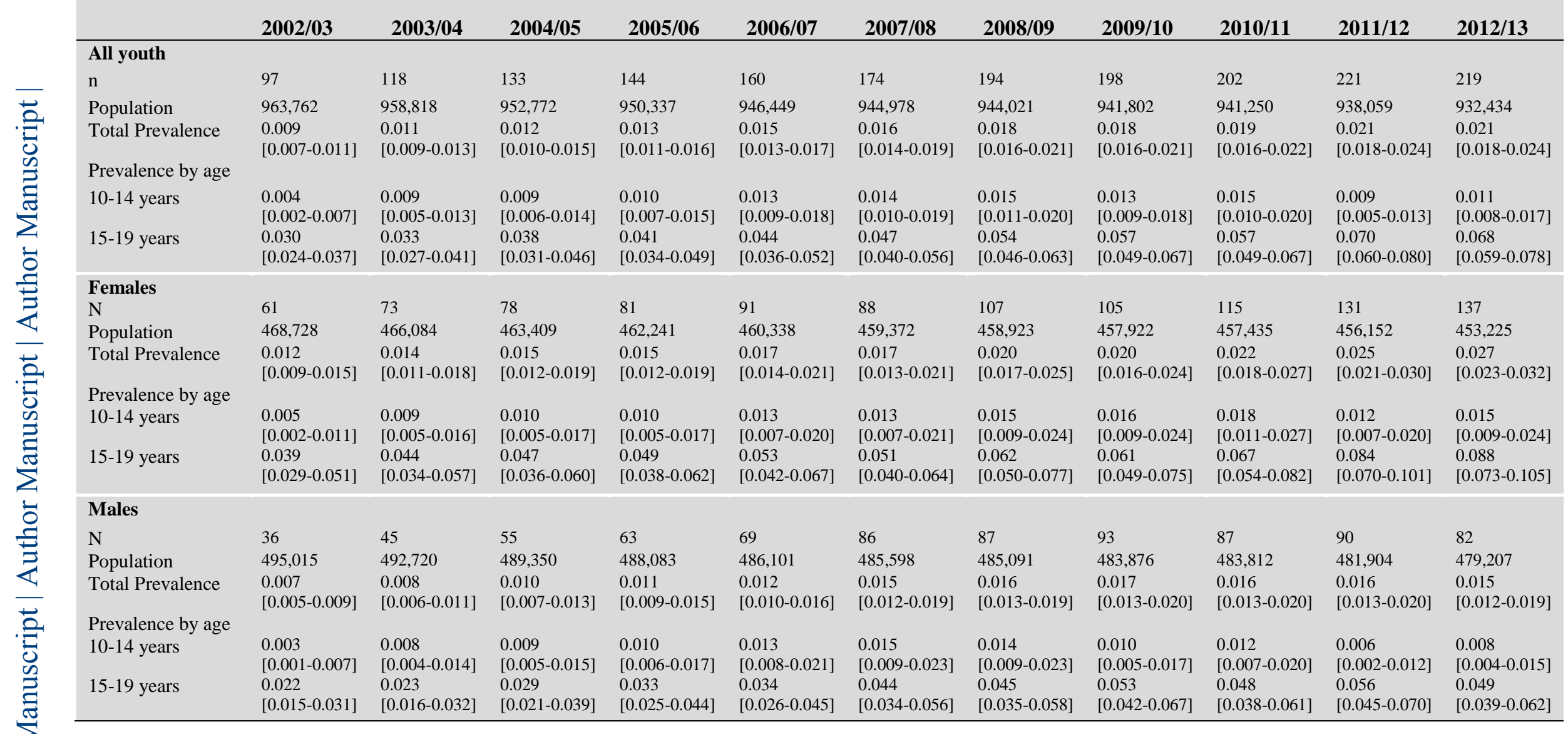

